\title{
Electric and magnetic characterization of $\mathrm{NbSe}_{2}$ single crystals: anisotropic superconducting fluctuations above $T_{C}$
}

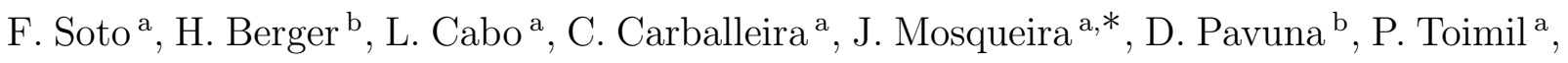 \\ F. Vidal ${ }^{\mathrm{a}}$ \\ ${ }^{a}$ LBTS, Departamento de Física da Materia Condensada, Universidade de Santiago de Compostela, E-15782 Spain \\ ${ }^{\mathrm{b}}$ Department of Physics, Ecole Politechnique Fédérale de Lausanne, CH-01015, Lausanne, Switzerland
}

\begin{abstract}
Electric and magnetic characterization of $\mathrm{NbSe}_{2}$ single crystals is first presented in detail. Then, some preliminary measurements of the fluctuation-diamagnetism (FD) above the transition temperature $T_{C}$ are presented. The moderate uniaxial anisotropy of this compound allowed us to observe the fluctuation effects for magnetic fields $H$ applied in the two main crystallographic orientations. The superconducting parameters resulting from the characterization suggest that it is possible to do a reliable analysis of the FD in terms of the Ginzburg-Landau (GL) theory.
\end{abstract}

Key words: Fluctuations, Magnetic properties, Binary compounds PACS: 74.40.+k, 74.20.De, 74.25.Ha, 74.70.Ad

The anisotropy of the FD above $T_{C}$ is still a relatively unexplored issue. The GL theory predicts that the fluctuation-induced magnetic susceptibility when $H$ is parallel to the crystallographic $a b$ planes is related to the one when $H \perp a b$ through $\Delta \chi_{\|}=\Delta \chi_{\perp} / \gamma^{2}$, where $\gamma$ is the anisotropy factor.[1] While most of low- $T_{C}$ superconductors are isotropic, the high $T_{C}$ 's are so anisotropic that $\Delta \chi_{\|}$ is practically unobservable (for the less anisotropic one, $\mathrm{YBa}_{2} \mathrm{Cu}_{3} \mathrm{O}_{7}$ with $\left.\gamma \approx 10, \Delta \chi_{\|} \approx 10^{-2} \Delta \chi_{\perp}\right)$. The moderate anisotropy of $\mathrm{NbSe}_{2}(\gamma \approx 3$, and then $\left.\Delta \chi_{\|} \gtrsim 10^{-1} \Delta \chi_{\perp}\right)$ makes this material an excellent test bed to explore anisotropy effects on the FD above $T_{C}$. Here we present a magnetic and electric characterization of $\mathrm{NbSe}_{2}$ single crystals. The resulting superconducting parameters will allow a reliable analysis of the FD measured above $T_{C}$.

The $\mathrm{NbSe}_{2}$ single crystals used in this work were synthesized by chemical transport method. The

\footnotetext{
* Corresponding author. e-mail: fmjesus@usc.es
}

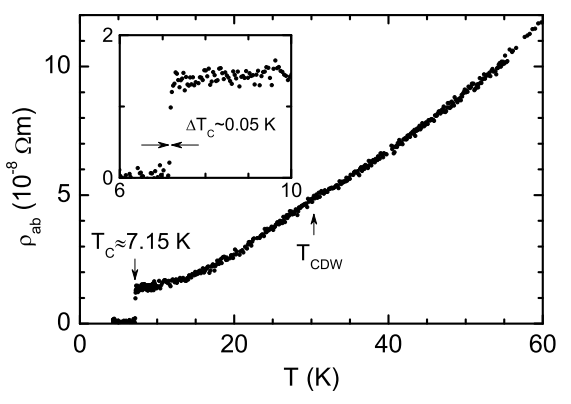

Fig. 1. Temperature dependence of the in-plane electrical resistivity. The kink at $\sim 30 \mathrm{~K}$ corresponds to a charge-density wave (CDW) transition.

transition temperature $T_{C}=7.15 \mathrm{~K}$ was determined from the temperature dependence of the in-plane electrical resistivity $\rho_{a b}$ (Fig. 1). This measurement was done in a $2 \times 1 \times 0.067 \mathrm{~mm}^{3}$ single crystal cut from one of the crystals used in the magnetization measurements, by using a van der Pauw contacts configuration. The ratio $\rho_{a b}(300 \mathrm{~K}) / \rho_{a b}(7.5 \mathrm{~K})=$ 


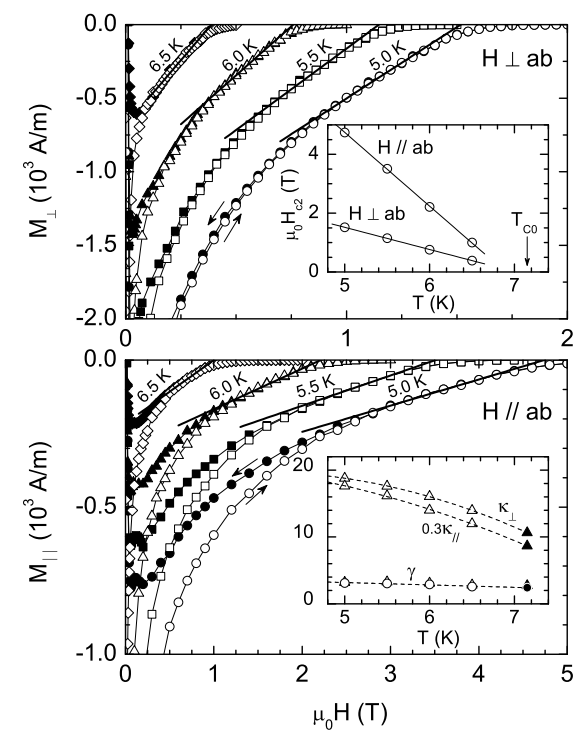

Fig. 2. $M(H)$ isotherms below $T_{C}$. The fits of the Abrikosov theory for the mixed state (thick lines) leads to the $H_{C 2}^{\perp, \|}$, $\kappa_{\perp, \|}$ and $\gamma$ values presented in the insets.

43 , is comparable to the ones found in the best crystals.[2] The in-plane mean free path $\ell_{a b} \approx 1830 \AA$ was obtained from the in-plane residual resistivity and from the carriers concentration, [3] by using a Drude-model relation. The resistivity anisotropy is found to be $\rho_{c} / \rho_{a b} \sim 60$, which leads to a mean free path in the $c$ direction of $\ell_{c} \approx 30 \AA$. The magnetization measurements were performed with a commercial (Quantum Design) SQUID magnetometer. In Fig. 2 we present $M(H)$ measurements at different temperatures below $T_{C}$ and with $H \perp a b$ and $H \| a b$. The thick lines in this figure are the best fit to the data points of the high-field mixed state magnetization of anisotropic superconductors [3]:

$M_{\perp, \|}(T, H) \approx\left[H-H_{C 2}^{\perp, \|}(T)\right] / 2 \beta_{A} \kappa_{\perp, \|}$

where $H_{C 2}^{\perp, \|}$ are the upper critical magnetic fields for $H \perp a b$ and $H \| a b$ respectively, $\kappa_{\perp}=\lambda_{a b} / \xi_{a b}$ and $\kappa_{\|}=\gamma \kappa_{\perp}$ are the corresponding GL parameters ( $\lambda_{a b}$ and $\xi_{a b}$ are, respectively, the in-plane magnetic penetration and coherence lengths) and $\beta_{A} \approx 1.16$. The resulting $H_{C 2}^{\perp, \|}$ and $\kappa_{\perp, \|}$ are presented in the insets of Fig. 2. As may be clearly seen, both upper critical fields are linear in $T$ in the temperature range studied, and extrapolate to $H_{C 2}^{\perp}(0) \approx 5.3 \mathrm{~T}$ and $H_{C 2}^{\|}(0) \approx 17.3 \mathrm{~T}$. By combining these values with the GL expressions $\mu_{0} H_{C 2}^{\perp}=\phi_{0} / 2 \pi \xi_{a b}^{2}$ and $\mu_{0} H_{C 2}^{\|}=\phi_{0} / 2 \pi \xi_{a b} \xi_{c}$, we obtained $\xi_{a b}(0)=78.8 \AA$

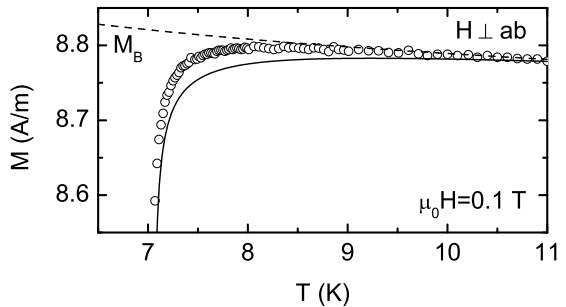

Fig. 3. $M(T)$ curve above $T_{C}$ for $H \perp a b . M_{B}$ is the normal-state contribution, and the solid line is the prediction of the anisotropic 3D-GL theory.

and $\xi_{c}(0)=24.1 \AA$. The GL parameters extrapolate to $\kappa_{\perp} \sim 11$ and respectively $\kappa_{\|} \sim 29$ at $T_{C}$. The anisotropy factor as calculated from $H_{C 2}^{\|} / H_{C 2}^{\perp}$ and from $\kappa_{\|} / \kappa_{\perp}$ tends to $\sim 2.4$ at $T_{C}$. The agreement of both $\gamma$ determinations is an important consistency check of the procedure used to obtain the $\mathrm{NbSe}_{2}$ superconducting parameters, which also agree with the values in the literature.[4]

A first example of the as-measured $M(T)$ above $T_{C}$ for $H \perp a b$ is presented in Fig. 3. The dashed line is the normal-state contribution, which was obtained by fitting a Curie-like function well above $T_{C}$ (for $\left.T>1.5 T_{C}\right)$. The solid line is the prediction of the anisotropic 3D-GL theory under a total-energy cutoff (see, e.g., Ref. [5]) evaluated with the above superconducting parameters. As may be clearly seen, the theory overestimates the experimental FD by a factor of $\sim 2$, which cannot be explained by the background determination uncertainty. On the contrary, when $H \| a b$ we found an excellent agreement between the theory and the experimental results. We suggest that these results may be explained in terms of the anisotropy of non-local electrodynamic effects on the FD. Also, the FD seems not to be appreciably affected by the CDW state appearing below $\sim 35 \mathrm{~K}$.

Supported by MEC and FEDER funds (MAT200404364), and by Xunta de Galicia (PGIDIT04TMT 206002PR). HB and DP acknowledge support by the EPFL and the Swiss National Science Foundation, and LC by a MEC FPU grant.

\section{References}

[1] D.E. Prober et al., Phys. Rev. B 15 (1977) 5245.

[2] H.N.S. Lee et al., J. App. Phys. 40 (1969) 602.

[3] V.G. Kogan, J. Clem, Phys. Rev. B 24 (1981) 2497.

[4] N. Toyota et al., J. Low. Temp. Phys. 25 (1976) 485. 
[5] J. Mosqueira et al., Phys. Rev. B 65 (2002) 174522. 